\section{MEDICTA 2003}

Vai realizar-se, entre 27 e 30 de Julho de 2003, no Departamento de Química da Faculdade de Ciências da Universidade do Porto, a 6th Mediterranean Conference on Calorimetry and Thermal
Analysis - MEDICTA 2003. Serão apresentadas várias comunicações incluídas nas seguintes secções: Termodinâmica Química, Soluções, Termoquímica de Compostos Orgânicos e Inorgânicos, Ciências Farmacêuticas, Alimentares e da Vida, Macromoléculas (Proteínas e outra moléculas biológicas), Materiais e Teoria, Novos Métodos e Calibração. Mais informações podem ser obtidas em http://medicta2003.fc.up.pt ou solicitadas para medicta2003@fc.up.pt.

\title{
NOTIPIÍRIN GFRM
}

\section{Actualidades Científicas}

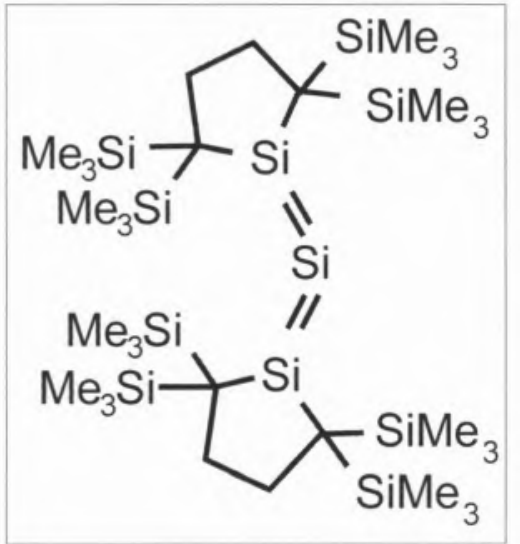

1.

As ligações múltiplas são usuais em química orgânica, em compostos como acetilenos, nitrilos, alenos e dióxido de carbono. Durante muito tempo, pensou-se que os homólogos mais pesados do carbono, como o silício e o estanho, não eram capazes de fazer ligações múltiplas porque as suas orbitais pi, que permitem a formação de ligaçōes múltiplas, sobrepõem-se pouco. Mas substituintes volumosos podem proteger tais duplas ligações, como o ilustra a síntese em 1981 da primeira molécula com dupla ligação silício-silício, o tetramesitildissileno. Desde então, quase 50 outros dissilenos foram sintetizados. Agora [1], um análogo estável dum composto aleno foi sintetizado, com uma unidade central $\mathrm{Si}=\mathrm{Si}=\mathrm{Si}$. Ao contrário do seu homólogo orgânico linear, o trissila-aleno é "dobrado", mas o composto é relativamente estável e deveria ser um começo útil para a sintese de novos materiais baseados no silício com estruturas electrónicas variadas.

\section{2.}

$\mathrm{O}$ diboreto de magnésio (MgB2) tem a temperatura de transição (Tc) mais alta conhecida para um metal supercondutor $(39 \mathrm{~K})$. A origem desta Tc anormalmente alta é objecto de estudos intensivos por dizer respeito quer à física fundamental da supercondutividade quer a potenciais aplicações industriais dos supercondutores. A espectroscopia de fotoemissão angular de alta resolução fornece agora [2] argumentos para apoiar as precedentes sugestões de que - MgB2 possui um novo mecanismo de supercondutividade com "duas bandas".

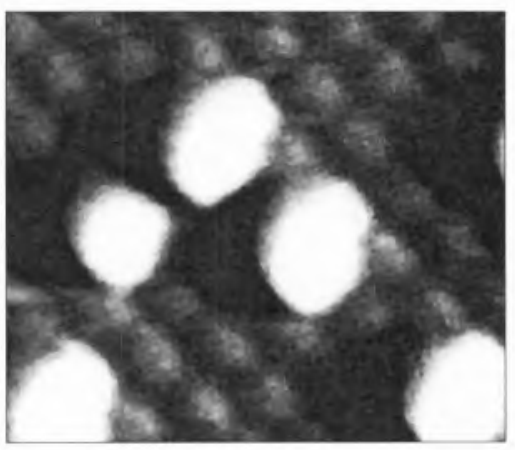

\section{3.}

Os sítios activos de catalisadores importantes como o paládio são estudados desde há muitos anos, mas ainda trazem surpresas. Mitsui et al. [3] apanharam agora o paládio em acção, num filme feito a partir de imagens de microscópio de varrimento por efeito túnel da formação de sítios activos que catali- sam a dissociação e a adsorção de moléculas de hidrogénio sobre o catalisador. Este acontecimento envolveu o abrandamento da reacção numa escala de segundos por arrefecimento a $65 \mathrm{~K}$, tornando possível a microscopia de resolução atómica. A descoberta surpreendente é que os sítios na superficie do catalisador com dois sítios de adsorção adjacentes e livres não proporcionam a adsorção dissociativa da H2. A catálise efectiva necessita sítios com um mínimo de três lacunas em proximidade imediata, não as duas que se esperaria que o dihidrogénio ocupasse.

\section{4.}

Uma publicação na revista Nature de 1949 iniciou um longo debate sobre a radioactividade natural do bismuto. 0 seu único isótopo natural 209Bi é habitualmente considerado como o isótopo estável mais pesado. De acordo com a teoria, no entanto, o 209Bi é metaestável e deveria decair por emissão de partículas a (cada uma constituída por dois protões e dois neutrões). Mas a probabilidade de decaimento é extremamente baixa e experiências levadas a cabo desde 1949 não conseguiram detectar qualquer radiação a. A tecnologia mais recente do bolómetro de cintilação ultrapassa as dificuldades anteriores: 0 209Bi é agora [4] oficialmente instável com um tempo de meia-vida de $2 \times 1019$ anos. $\mathrm{O}$ isótopo pesado seguinte mais estável, o 208Pb, tem uma estrutura nuclear muito estável. 0 seu decaimento pode, portanto nunca vir a ser observado. Embora, dentro de 55 anos, quem sabe? 
Referências

[1] S. Ishida, T. Iwamoto, C. Kabuto, M. Kira, Nature 421 (2003) 725.

[2] S. Souma, Y. Machida, T. Sato, T. Takahashi, H. Matsui, S.-C. Wang, H.
Ding, A. Kaminski, J.C. Campuzano, S. Sasaki, K. Kadowaki, Nature 423 (2003) 65.

[3] T. Mitsui, M.K. Rose, E. Fomin, D.F. Ogletree, M. Salmeron, Nature 422 (2003) 705.
[4] P. de Marcillac, N. Coron, G. Dam-

bier, J. Leblanc, J.-P. Moalic, Nature 422 (2003) 876.

Olivier Pellegrino

Revisão de A. M. Botellho do rego

\section{Simpósio Internacional de Feixes Moleculares em Lisboa}

A Faculdade de Ciências e Tecnologia da Universidade Nova de Lisboa organiza, de 8 a 13 de Junho, no Altis Park Hotel, em Lisboa, o XX International Symposium on Molecular Beams, um dos mais importantes encontros mundiais nas áreas da Química-Física e da Física Atómica e Molecular.
Este evento bienal, que completa agora 40 anos de existência, vai realizar-se pela primeira vez no nosso país. Conta com o apoio da FCT/UNL e da Fundação para a Ciência e Tenologia, entre outras instituições.

Em discussão estarão fundamentalmente os seguintes temas: "Feixes Moleculares e Ciência à Nano-Escala"; "Colisões Atómicas e Moleculares"; "Fotodinâmica e Es- pectroscopia Laser"; "Dinâmica e Estrutura de Agregados Atómicos e Moleculares" e "Interacções Feixe-Superfície".

São vários os oradores convidados que têm contribuído decisivamente para o desenvolvimento destas áreas científicas, como se pode constatar na página http://events.fct.unl.pt/molbeam.

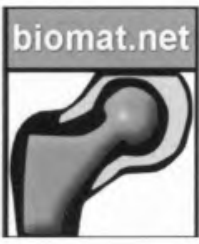

\section{Biomat.net (Biomaterials Network)}

A Biomaterials Network ou Biomat.net é uma página web dedicada à ciência dos biomateriais, criada por investigadores do INEB - Instituto de Engenharia Biomédica (Universidade do Porto) - , que comemorou o seu quinto aniversário em Fevereiro de 2003. A Biomat.net é actualmente a mais importante ferramenta de interligação entre a comunidade de biomateriais mundial.

A Biomat.net constitui uma colecção original de links WWW e ainda uma fonte de informação interactiva, onde os utilizadores podem participar activamente e comunicar uns com os outros. Esta página é mantida por um painel editorial internacional e supervisionada por um painel científico internacional, e constitui um serviço público gratuito, cujos principais objectivos consistem em:

- Proporcionar um recurso de comunicação de biomateriais organizado e significativo para cientistas, investigadores, para a comunidade empresarial, governos, meios académicos e público em geral;

- Actuar como um centro de divulgação de recursos da internet, organizaçōes, iniciativas educacionais, actividades de investigação, eventos científicos, artigos, jornais, livros, desenvolvimentos industriais, empregos e qualquer outra iniciativa relacionada com a ciência dos biomateriais.

A Biomat.net tornou-se num relevante veículo de informação para os investigadores das áreas dos biomateriais e engenharia de tecidos e num importante portal de biomateriais, onde informaçāo actualizada pode ser encontrada. Foi considerada entre os sites de topo nesta área por várias e distintas fontes, nomeadamente: Institute for Scientific Information (ISI)/Current Web Contents (2002), Science (2001), Cell \& Molecular Biology Online (2001), BioMedNet (2001), Bioquimica.online.pt (2001), European Medical Device Manufacturer (2000), Medical Device Link (2000), Medical Device \& Diagnostic Industry (1998).
A Biomat.net foi criada e é mantida por uma equipa internacional de investigadores em biomateriais, o que em muito contribui para a precisão dos seus conteúdos científicos. As suas funcionalidades incluem uma newsletter mensal (com notícias relevantes), uma base de dados de empregos, uma ferramenta permitindo troca de informação entre investigadores, assim como uma lista actualizada de encontros científicos e conferências, organizações, instituições educacionais e de investigação, jornais, livros, artigos e organizações industriais. Também disponibiliza uma selecção mensal de Top 5 sites. Novos websites relevantes são adicionados todos os meses a todas as secções de links. A secção de oferta e procura de emprego, que é actualizada diariamente, já permitiu a obtenção de empregos ou de funcionários a diversos candidatos e instituições de todo o mundo. A Biomat.net também apoia e patrocina eventos científicos, sobretudo através da divulgação de informação. A Biomat.net patrocinou já 126 eventos científicos. Outro serviço útil que disponibiliza incluí revisões de livros recentes efectuada por especialistas na área. 
Durante 2002 a Biomat.net continuou o seu crescimento e estabeleceu-se como a principal fonte de informação na internet na área dos biomateriais. Em Agosto o Institute for Scientific Information (ISI) seleccionou a Biomat.net para o Current Web Contents, o que significa que tudo que for publicado na Biomat.net se torna disponivel para uma audiência mundial através desta importante base de dados internacional.

A Biomat.net contabiliza mais de 2800 membros oriundos de cerca de 85 paí- ses, e de mais de 1000 instituições. Em 2002, 842 novos investigadores tornaram-se membros. Os visitantes aumentaram 26\% em 2002, provenientes de mais de 120 paises. Respectivamente, $44,5 \%$ e $28,1 \%$ deles acederam à Biomat.net da Europa e América do Norte. $62.2 \%$ dos membros pertencem ao meio académico, $20.7 \%$ a indústrias, $6.1 \%$ a instituições governamentais, $4.0 \%$ a hospitais, $1.0 \%$ a editoras e $5.8 \%$ são de outras actividades não especificadas.
No presente, a Biomat.net é essencialmente dirigida a especialistas, apesar de alguma informação para o público em geral estar disponivel, tal como artigos publicados em revistas de interesse geral. Futuras funcionalidades estão a ser desenvolvidas, fornecendo informação acerca de biomaterais concebida especialmente para o público em geral.

Abril de 2003

Pedro L. Granja, Editor-in-chief José Paulo Pereira, Editor

\section{Relatório da IUPAC - \\ Substâncias Naturais e Artificiais Relacionadas com a Saúde Humana}

Para distribuição imediata, 22 de Abril de 2003

Num artigo publicado recentemente na revista Pure and Applied Chemistry, o "Subcommittee on Medicinal Chemistry and Drug Development" da IUPAC refere que as substâncias naturais e os compostos sintéticos são complementares para a produção de novos medicamentos. No entanto, no público em geral, é frequente surgirem questões que põem em confronto compostos naturais ou sintéticos, em tópicos que vão desde as plantas medicinais ao arsenal da guerra química.

$O$ facto de um composto ser de origem natural ou sintética é irrelevante.

Os compostos naturais têm tipicamente estruturas complexas que não são fáceis de obter sinteticamente. No entanto, devem por vezes ser modificados para aumentar as suas propriedades medicinais desejáveis ou diminuir as tóxicas. Há, porém, muitos agentes medicinais úteis obtidos por síntese total. Assim, para o desenvolvimento de um novo medicamento para uma doença particular pode existir a opção de partir de uma substância que ocorre naturalmente ou de utilizar matérias primas mais básicas.
Por exemplo, os agentes contra o cancro constituem uma classe terapêutica particular para a qual os produtos naturais têm sido importantes. Devido à suas estruturas complexas, são difíceis de preparar sinteticamente e ainda são obtidos a partir de produtos naturais.

Muitos antibióticos como a penicilina e eritromicina são obtidos a partir de substâncias naturais modificadas sinteticamente a fim de produzirem os efeitos desejados. Outros são obtidos por síntese total. Todos os medicamentos usados para tratar o HIV e as infecções por herpes são produzidos sinteticamente; o uso de produtos naturais para tratar doenças virais tem tido um sucesso muito limitado.

Os medicamentos à base de plantas constituem uma classe à parte. São geralmente misturas complexas de vários componentes que não estão muito bem caracterizadas ou compreendidas. As condições em que uma planta cresce ou os processos de extracção podem traduzir-se numa variação do produto obtido dependente da origem daquela planta particular. Como a composição pode variar, o mesmo pode acontecer com a sua eficácia terapêutica ou com os efeitos secundários. Embora as plantas medicinais sejam conhecidas de há muito, a compreensão da sua acção ainda está na infância. Investigações futuras podem proporcionar benefícios significativos para a descoberta de novos medicamentos.

As vitaminas, essenciais para uma nutrição adequada, podem ser obtidas natural ou sinteticamente. Apesar de uma considerável promoção da ideia que as fontes naturais de vitaminas são mais benéficas que as produzidas por síntese, muitas vitaminas, como a vitamina C, são sintetizadas de uma forma idêntica à da natureza.

\section{A toxicidade de certas substâncias e a} sua utilização potencial no arsenal de guerra química tem recebido recentemente uma grande atenção. A toxicidade de um composto não é função da sua origem natural ou sintética. Substâncias extremamente tóxicas como a ricina e a toxina botulínica podem ser obtidas de fontes naturais. Apesar de uma elevadíssima toxicidade, alguns compostos têm estado a ser usados em doses mínimas com efeitos terapêuticos prometedores.

A segurança e eficácia de uma molécula de uma droga é função da sua estrutura e não da sua origem. A estrutura molecular define as suas interacções com outras moléculas do organismo e é a razão pela qual a substância apresenta actividades desejáveis ou indesejáveis. 0 facto de o composto ter origem natural ou sintética é irrelevante.

Relatório oficial escrito por Dr. Thomas Perun, Professor Paul Erhardt, e Dr. 
James McAlpine. Para ver o relatório na integra, visite http://www.iupac.org/publications/pac/2002/7410/7410×1957.h tml

A IUPAC foi fundada em 1919 por químicos académicos e da indústria. Durante cerca de 85 anos a União tem conseguido promover a comunicação a nível mundial das ciências químicas e unir a química industrial, a académica e a do sector público na utilização de uma linguagem comum. A IUPAC é reconhecida como a autoridade mundial em nomenclatura química, terminologia, métodos estandardizados de medida, massas atómicas e muitos outros dados avaliados criticamente. Há mais infor- mação disponivel sobre a IUPAC e as suas actividades em www.iupac.org.

Para resposta a qualquer questão contacte Laura Abernathy, IUPAC Communications Specialist, Laura@iupac.org

Tradução: Elisa Maia

Professora auxiliar (aposentada) do Departamento de Química da FCUL elisamaia@netcabo.pt

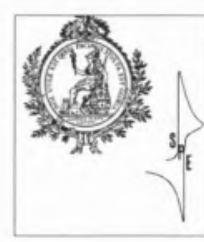

\section{New Trends in Molecular} Electrochemistry and XII Meeting of the Portuguese

\section{Electrochemical Society}

O XII Encontro da Sociedade Portuguesa de Electroquímica e o Congresso sobre New Trends in Molecular Electrochemistry terão lugar de 16 a 20 de Setembro do corrente ano, na Academia das Ciências de Lisboa.

As respectivas fichas de inscrição e submissão dos resumos dos trabalhos a

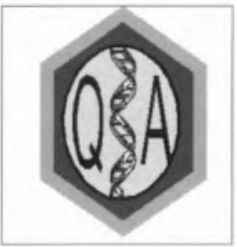

Nos dias 16 e 17 de Outubro do corrente ano realizar-se-á no Campus da Faculdade de Ciências e Tecnologia, da Universidade Nova de Lisboa, 0 I FóRUM DA QUÍMICA subordinado ao tema " Três Faces da Química: Investigação, Indústria e Ensino" organizado pelos finalistas da licenciatura de Quími- apresentar, bem como os demais detaIhes, encontram-se na página destes Encontros:

\section{http://dequim.ist.utl.pt/acl-spe}

Qualquer informação adicional poderá ser obtida do Prof. Armando Pombeiro ou da Dr. ${ }^{\text {a }}$ Fátima Guedes da Silva:

pombeiro@ist.utl.pt ou fatima.guedes@ist.utl.pt

Centro de Química Estrutural, Complexo I, Instituto Superior Técnico,

Av. Rovisco Pais 1049-001 Lisboa, Portugal Fax: +351-218464455/7 Tel.: +351-218419225/235/237

ca Aplicada (ramos química orgânica e biotecnologia).

Com a concretização deste evento, pretende-se identificar e debater os vectores de desenvolvimento nas áreas de química orgânica e biotecnologia a nível nacional.

Para mais informações, consulte www. dq.fct.unl.pt/forum_da_quimica

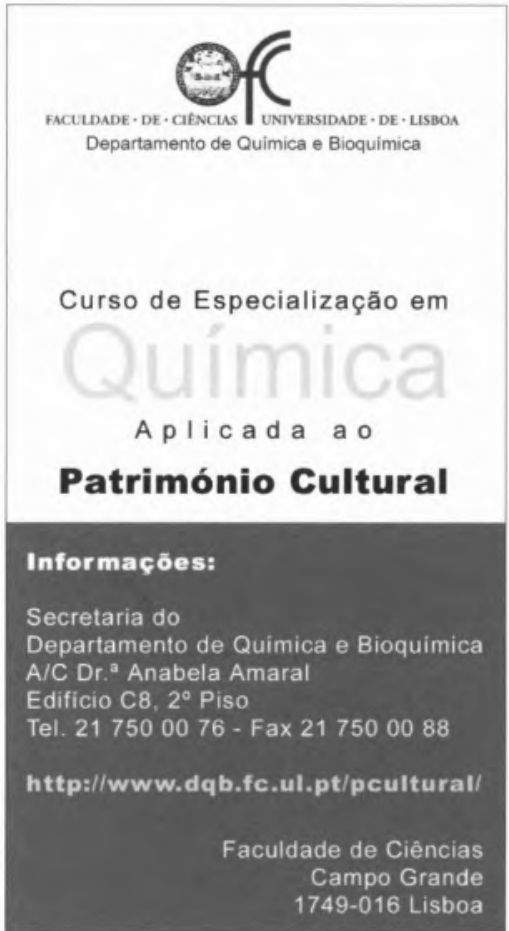

Dear colleagues and friends,

The $5^{\text {th }}$ meeting of EFEPR (European Federation of EPR groups) is coming soon and the deadline for abstract (for poster and oral presentations) submission is June 7 th. We have an excellent program and we look forward to a stimulating meeting. There are slots for oral presentations to be selected from the abstracts and we would like to have at least some of them filled up by the young, bright students in our field. All the details can be found in the website http://dequim.ist.utl.pt/EFEPR/. We advise you to make your hotel reservations as soon as possible since the meeting is during the hot tourist season and the earlier you book the better.

Looking forward to seeing you in Lisbon Joao Paulo Telo and Daniella Goldfarb
(7) $3^{\text {d Intemational Congress }}$ First announcement and call for papers

Pigmentsinfood more than colours..

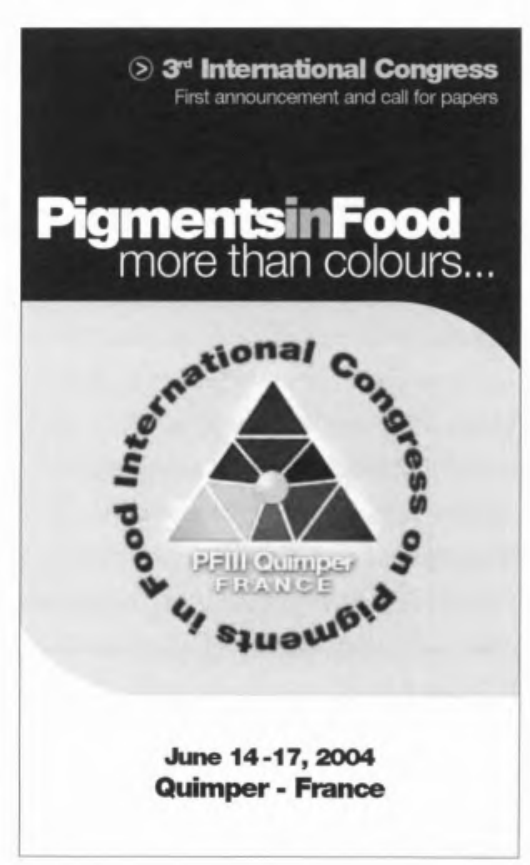

